ARTICLE

https://doi.org/10.1038/s41467-019-10867-w

\title{
Orthogonal regulation of phytochrome B abundance by stress-specific plastidial retrograde signaling metabolite
}

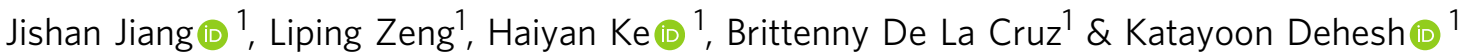

\begin{abstract}
Plant survival necessitates constant monitoring of fluctuating light and balancing growth demands with adaptive responses, tasks mediated via interconnected sensing and signaling networks. Photoreceptor phytochrome B (phyB) and plastidial retrograde signaling metabolite methylerythritol cyclodiphosphate (MEcPP) are evolutionarily conserved sensing and signaling components eliciting responses through unknown connection(s). Here, via a suppressor screen, we identify two phyB mutant alleles that revert the dwarf and high salicylic acid phenotypes of the high MEcPP containing mutant ceh1. Biochemical analyses show high phyB protein levels in MEcPP-accumulating plants resulting from reduced expression of phyB antagonists and decreased auxin levels. We show that auxin treatment negatively regulates phyB abundance. Additional studies identify CAMTA3, a MEcPP-activated calcium-dependent transcriptional regulator, as critical for maintaining phyB abundance. These studies provide insights into biological organization fundamentals whereby a signal from a single plastidial metabolite is transduced into an ensemble of regulatory networks controlling the abundance of phyB, positioning plastids at the information apex directing adaptive responses.
\end{abstract}

\footnotetext{
${ }^{1}$ Department of Botany and Plant Sciences, Institute of Integrative Genome Biology, University of California, Riverside, CA 92521, USA. Correspondence and requests for materials should be addressed to K.D. (email: kdehesh@ucr.edu)
} 
$\mathrm{P}$ lant survival is dependent on light perception, required for photosynthetic activities and elicitation of biochemical and molecular modifications key to consequential adjustment of growth and development in a constantly changing environment. Phytochromes (phys) are a family of cytosolic photoreceptors (phyA to phyE) with varying extent of physiological functions, thus superimposing a layer of specialization on a foundation of partially overlapping functions ${ }^{1-4}$. Upon perception of red light, phys are activated and translocated to the nucleus, where they initiate and transduce photomorphogenesis signaling cascade. The main photoreceptor mediating photomorphogenesis in red light, however, is phyB. Red light activated phyB is imported into the nucleus and forms phyB-containing nuclear bodies (phyB$\mathrm{NBs})^{3,5}$. Formation of phyB-NBs depends on binding to and sequestration of the basic helix-loop-helix (bHLH) transcription factors, Phytochrome Interacting Factor 1 (PIF1), PIF3, PIF4, PIF5, and PIF76,7. This binding facilitates phosphorylation and degradation of PIFs by the $26 \mathrm{~S}$ proteasome and reduces their capacity to bind their DNA targets to initiate responses $6,8,9$.

Shade avoidance response (SAR) is a central physiological and developmental response to the light signals resulting from changes in the ratio of red (R):far-red (FR) light caused by neighboring vegetation leading to among others enhanced stem and petiole growth as a mechanism attempting to reach an strata with more available light ${ }^{10-12}$. Although several phys contribute to SAR, phyB plays a particularly prominent role, as evidenced by the display of classical SAR phenotypes in phyB mutants ${ }^{10,11}$. The SAR-mediated enhanced growth can also be simulated by exogenous application of auxin as well as through genetic manipulations such as generation of lines with increased auxin levels $^{13-17}$. It is well established that PIF4, PIF5, and PIF7 play a major role, whereas PIF1 and PIF3 assume a minor role in mediating SAR, and additional evidence indeed illustrate that PIFs target promoter elements of multiple auxin biosynthetic and signal transduction genes ${ }^{8,18-20}$.

The overall function of phys in transcriptional regulation conveys exquisite light control of photosynthetic machinery in chloroplast, followed by consequential physiological responses. However, the success of these biological processes depends on tight coordination and dynamic alignment of cellular responses to light perception through activation of signal transduction pathways essential for fine-tuning of inter-organellar communication, specifically through chloroplast-to-nucleus signaling, a process called retrograde signaling ${ }^{21}$.

Our search for identification of a stress specific retrograde signal, led to the discovery of the plastid-derived metabolite 2-Cmethyl-d-erythritol 2,4-cyclodiphosphate (MEcPP), that functions both as a precursor of isoprenoids produced by the conserved and essential plastidial methylerythritol phosphate (MEP) pathway, and as a stress-specific retrograde signaling metabolite that communicates plastidial perturbations to the nucleus ${ }^{22}$. This discovery was founded on a genetic screen that led to the isolation of a mutant line designated ceh1, for constitutive expression of Hydroperoxide Lyase (HPL), an otherwise stress-inducible nuclear gene encoding a plastidial enzyme in the HPL branch of the oxylipin pathway ${ }^{22}$. This mutation results in accumulation of high MEcPP levels and the consequent growth retardation in concert with induction of selected stress-responsive nuclear genes and their respective metabolites. Among the nuclear genes with enhanced expression level in ceh1 is isochorismate synthase 1 (ICS1), a stress-inducible nuclear gene encoding a key plastidial enzyme in the salicylic acid (SA) biosynthetic pathway ${ }^{22,23}$. Furthermore, we established that MEcPP transduces signals to activate a core set of general stress response genes through induction of a transcriptionally centered stress hub, activated predominantly by the function of a member of the calmodulin- binding transcriptional activator family, CAMTA $3^{24}$. In addition, we demonstrated that MEcPP controls adaptive growth by modulating auxin levels and distribution patterns in response to stress $^{25}$.

In this study, to identify the genetic components of MEcPP signal transduction pathway we report the results of a suppressor screen for revertants with fully or partially abolished ceh1 phenotypes in spite of high MEcPP levels. This approach led to the identification of two independent $p h y B$ mutant alleles that notably reverted high SA levels and dwarf stature phenotypes of the ceh1 mutant. Subsequent analyses of these mutant lines identified the network of MEcPP-mediated signaling cascade that alter phyB protein level and uncovered the role of auxin and CAMTA3 in maintaining phyB abundance. Moreover, pharmacological approaches illustrated that the likely function of CAMTA3 is to stabilize phyB protein level through impairment of proteasome-mediated degradation machinery. We conclude that phyB is a component of MEcPP-signal transduction pathway and that MEcPP-mediated regulation of phyB level is multifaceted. By extension this sheds light on the links between the two evolutionarily conserved sensing and signaling cascades essential for adjustment of growth and development in a constantly changing environment.

\section{Results}

PhyB is a component of MEcPP signal transduction pathway. To identify the genetic components of the plastidial retrograde signal transduction pathway, we mutagenized the high MEcPPaccumulating ceh1 in search of revertants that despite high MEcPP levels display full or partial recovery of the mutant dwarf stature with either no and/or reduced expression of HPL and/or the ICS1-derived product SA. This led to identification of two independent revertants (41-16 and 56-6) with classical SAR phenotypes, namely seedlings with long hypocotyls and long petioles $^{10-12}$, and overall a larger statue than that of the ceh1 mutant, albeit still smaller than the parent wild-type (P) seedlings (Fig. 1a, b and Supplementary Fig. 1a). Furthermore, measurements of MEcPP show a $\sim 50 \%$ reduction of the metabolite levels in the revertants as compared with the ceh1, yet with $\sim 200$-fold higher levels than that of the $\mathrm{P}$ seedlings (Fig. 1c). Following analyses showed notable $(\sim 70 \%)$ reduction in SA levels of the revertants as compared with ceh1 (Fig. 1d). Subsequent sequencing analyses identified two different single non-sense mutations in the $P H Y B$ resulting in premature terminations of the protein in revertants (Fig. 1e). Complementation studies using the wild type $P H Y B$ coding region under the control of the native promoter reestablished the ceh1 mutant phenotypes in the revertants, and conversely the double mutant (ceh1/phyB-9) generated from crosses between ceh 1 and a phyB null mutant (phyB-9) mimicked the growth phenotypes and the SA levels of the revertants (Fig. $1 \mathrm{a}-\mathrm{d}$ ). The data verifies revertants as $p h y B$ mutant alleles.

To examine phyB general and/or specific function in MEcPPmediated signal transduction pathway, we also compared expression of HPL, a stress-inducible gene constitutively expressed in the ceh1, in aforementioned genotypes. Comparable HPL expression levels in ceh1 and ceh1/phyB-9 seedlings excluded the involvement of phyB in the MEcPP-mediated induction of HPL (Supplementary Fig. 1b).

The data collectively establishes phyB as a component of $\mathrm{MEcPP}$ signal transduction pathway involved in regulation of growth and SA level, but not in the induction of HPL expression.

PhyB protein abundance in the MEcPP-accumulating lines. To address a potential transcriptional/posttranscriptional alteration of phyB in MEcPP accumulating plants, we initially examined the 
ceh1/

P ceh1 41-16 56-6 CP phyB-9

a

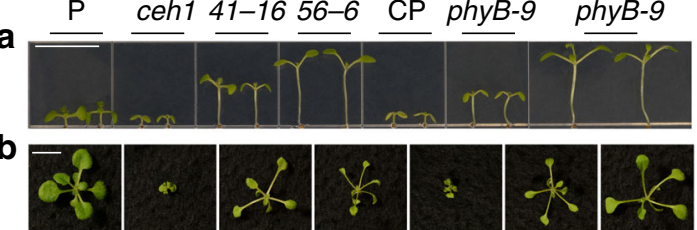

c
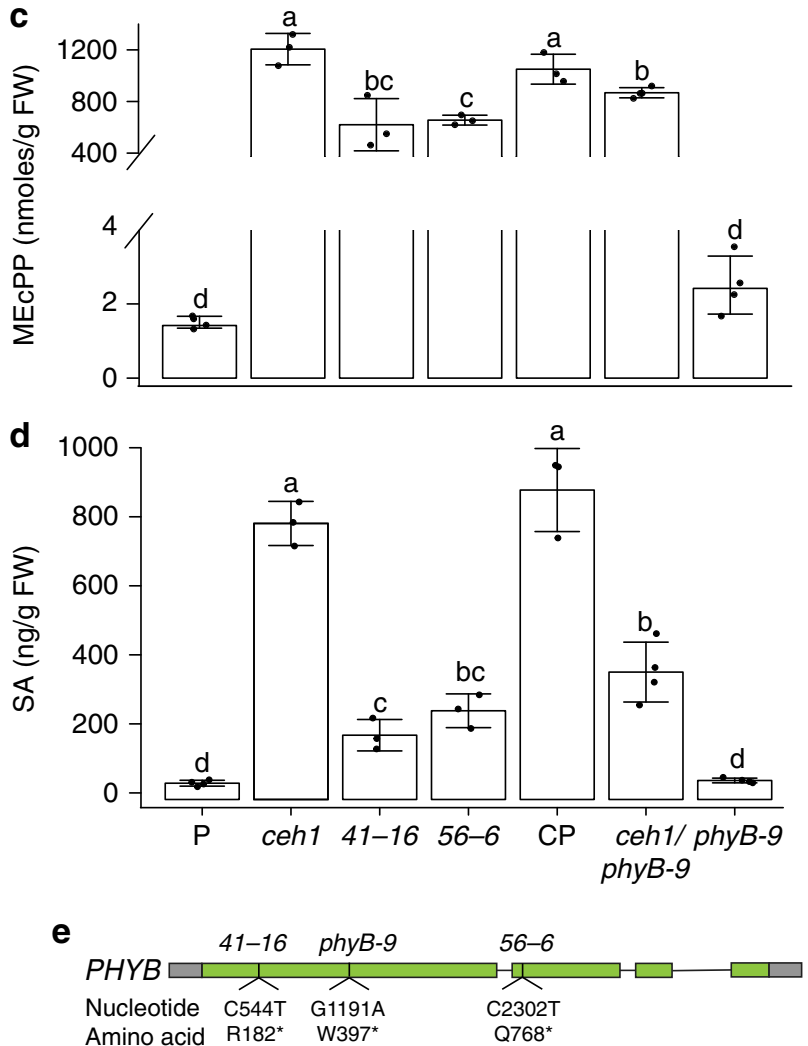

Fig. 1 PhyB is a component of MEcPP signal transduction pathway. a, b Representative images of 1-week-old and 2-week-old P, ceh1, 41-16, 56-6, complementation line (CP), ceh1/phyB-9, and phyB-9 seedlings grown in long-day (LD; $16 \mathrm{~h}$ light/8 $\mathrm{h}$ dark), respectively. c MEcPP measurements of 2-week-old seedlings of aforementioned genotypes. The white line indicates a change of scale on the $Y$ axis. d Measurements of Salicylic Acid (SA) levels of samples used in (c). e Schematic illustration of PHYB gene. Green boxes represent exons, gray boxes represent UTRs, the actual nucleotide and amino acid changes of the mutants are displayed underneath. Data are mean $\pm S D$, each genotype with at least three biological replicates. Letters above bars indicate significant differences determined by Tukey's HSD method $(P<0.05)$. Scale bar: $1 \mathrm{~cm}$

PHYB transcript levels in ceh1 seedlings. Similar $P H Y B$ transcript levels in ceh1 and $\mathrm{P}$ plants excludes a MEcPP-mediated transcriptional regulation of the photoreceptor (Fig. 2a). Next, we examined phyB protein levels by immunoblot analyses of total proteins extracted from dark and long-day (LD; $16 \mathrm{~h}$ light $/ 8 \mathrm{~h}$ dark) grown $\mathrm{P}$ and ceh1 seedlings. These results showed similarly high phyB protein levels in the dark grown $\mathrm{P}$ and ceh1 genotypes (Fig. 2b). Intriguingly, we observed the expected reduction in phyB protein of $\mathrm{P}$ seedlings grown under LD whereas, under the same growth conditions the ceh1 seedlings maintained comparable levels of phyB protein to that present in the dark grown seedlings (Fig. 2b).

Total protein extract represents biologically active (nuclear) and biologically inactive (cytosolic) forms of phyB. To differentiate between the two states of phyB, we examined phyB protein abundance of nuclear protein extracts by immunoblot analyses. The outcome mimicked the total protein extract data, and further confirmed the presence of higher phyB protein levels in the nuclear extract of LD grown ceh1 as compared with $\mathrm{P}$ (Fig. 2c).

To establish a potential link between MEcPP and phyB protein abundance, we performed immunoblot analyses on protein extracts of DEX-inducible MEcPP accumulating line (HDSi) and the control $\mathrm{P}$ plants, before and at different hours post induction. The increased phyB levels in the induced HDSi seedlings establishes a correlation between accumulation of MEcPP and enhanced phyB protein abundance (Fig. 2d and Supplementary Fig. 1c).

In addition to $\mathrm{MEcPP}$, ceh1 mutant accumulates substantial amount of the defense hormone, salicylic acid $(\mathrm{SA})^{22,26}$. The reported phyB role in SA accumulation and signaling ${ }^{27}$ prompted us to examine the potential reciprocal role of this defense hormone in regulation phyB protein abundance, by employing the previously generated SA deficient double mutant line, ceh1/eds $16^{22}$. Immunoblot analyses on proteins extracts of $\mathrm{P}$, ceh1, eds16-1, and ceh1/eds16-1 clearly demonstrate comparable phyB protein levels in ceh1 and ceh1/eds16 (Supplementary Fig. 2a). This confirms that phyB abundance in the ceh1 mutant is SA-independent.

Next, we tested biological ramification of over-accumulation of phyB in ceh1 by fluence response studies carried out by measurements of hypocotyl lengths of seedlings grown in dark and continuous red-light $(\mathrm{Rc})$ with increasing fluence rate (light intensity) (Fig. 3a). Dark grown $\mathrm{P}$ and ceh1 seedlings exhibit similar hypocotyl lengths, whereas under Rc these genotypes display differentially reduced hypocotyl growth. The progressively shorter hypocotyl length of ceh1 with increasing fluence rate of red-light as compared with the $\mathrm{P}$ seedlings suggests photosensory hypersensitivity of ceh1 to Rc, most likely due to enhanced phyB protein abundance (Fig. 3a). This notion was further tested by examining hypocotyl length of seedlings grown in continuous white light (CL), and short-day (SD; $8 \mathrm{~h}$ light/16 h dark) conditions. The statistically significant lower relative SD/CL hypocotyl length in ceh1 as compared with $\mathrm{P}$ implies the presence of a more active (Pfr) form of phyB in the ceh1 relative to the relaxed (Pr) form (Fig. 3b).

This data collectively establishes the tight correlation between MEcPP accumulation and enhanced phyB protein abundance, ultimately resulting in photo-sensory hypersensitivity of ceh1 to red light.

PIF4 and PIF5 overexpression mimic revertants phenotypes. PIFs are known antagonist of phys, and established agonists of SAR response ${ }^{8,10}$. Amongst the seven PIF family members, PIF4 and PIF5 have been shown unequivocally to mediate this response ${ }^{8,10,14}$. Moreover, phyB-PIF interaction reciprocally induces phyB degradation, in a mutually-negative, feedback-loop configuration $^{9,28}$. To examine the potential role of PIFs in the ceh1 transduction pathway, we examined expression levels of PIF1, 3, 4, and 5, of which, PIF4 and PIF5 expressions were reduced in ceh1 as compared with $\mathrm{P}$ (Fig. 4a and Supplementary Fig. 2b). The slight reduction $(25-30 \%)$ in the PIF4 and 5 expression levels led us to genetically assess their involvement in the MEcPP-mediated signaling cascade by generating ceh1 overexpressing PIF4 and PIF5 lines. Subsequent analyses display partial recovery of ceh1 dwarf phenotype in the overexpresser backgrounds, with more pronounced growth in ceh1/PIF5-OX compared with ceh1/PIF4-OX seedlings (Fig. 4b). This differential growth correlates well with the respective MEcPP content, 


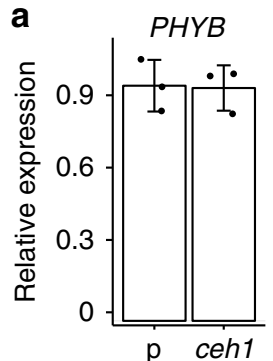

b $\mathrm{kD}$
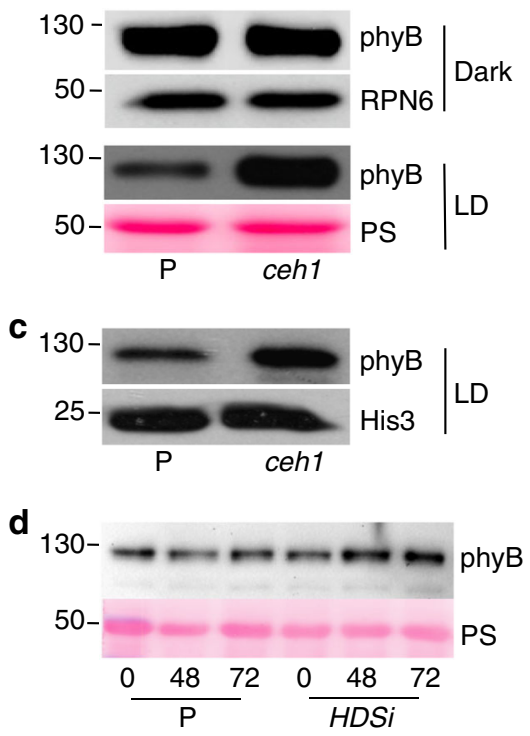

Fig. 2 PhyB protein abundance in MEcPP-accumulating lines. a PHYB expression level is not altered in ceh1 as compared with $\mathrm{P}$. Total RNAs were isolated from 7-day-old seedlings of $\mathrm{P}$ and ceh1 grown under long-day (LD; $16 \mathrm{~h}$ light/ $8 \mathrm{~h}$ dark) condition and subjected to qRT-PCR analysis. The mRNA levels of PHYB gene was normalized to the levels of At4g26410 (M3E9). Data are mean \pm SD of three biological replicates and three technical replicates. Two-tailed Student's $t$ tests confirms no significant differences of PHYB expressions between $\mathrm{P}$ and ceh1. $\mathbf{b}$ PhyB protein abundance in dark and LD grown $P$ and ceh1 seedlings. Total proteins extracted from 7-day-old dark and LD grown $\mathrm{P}$ and ceh1 seedlings were subjected to immunoblot analyses using phyB antibody. Immunoblot using RPN6 antibody in concert with Ponceau S (PS) staining were used as loading controls for dark and LD grown seedlings, respectively. C Immunoblot analyses of phyB protein levels in nuclear protein extracts from LD grown 7-day-old $P$ and ceh1 seedlings. Blots probed with Histone 3 (His 3) antibody show equal loading of nuclear proteins. $\mathbf{d}$ PhyB protein levels in 7-day-old P and DEX-inducible MEcPP-accumulating HDSi seedlings before (0) and hours (48 and 72) post induction. Ponceau S (PS) staining was used as the protein loading control

namely the presence of $\sim 20 \%$ less MEcPP in ceh1/PIF5-OX than in ceh1 or ceh1/PIF4-OX lines (Fig. 4c). Next, we analyzed SA levels and determined a $\sim 60-65 \%$ reduction in the SA content of ceh1/PIF4 and 5 overexpresser lines compared with the ceh1 mutant background (Fig. 4d).

The recovery of ceh1 dwarf phenotype in PIF4 and PIF5 overexpressing seedlings led us to examine the phyB protein levels in the respective lines by immunoblot analyses. The data show the expected reduced phyB abundance in ceh1/PIF4-OX and ceh1/PIF5-OX as compared with ceh1, and the expected lower abundance in both OX seedlings relative to P plants. It is of note that phyB relative abundance in ceh1/PIF4-OX is higher than that
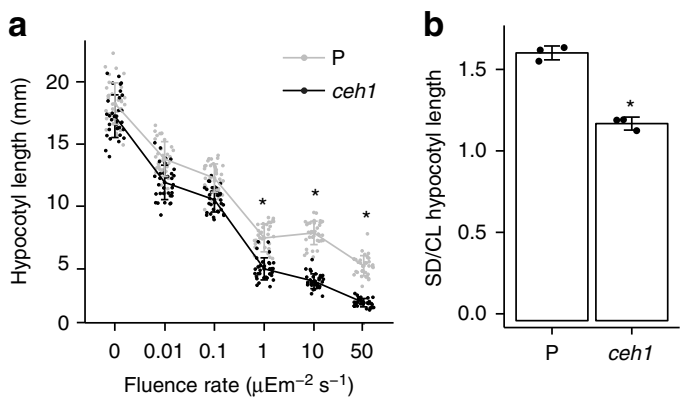

Fig. 3 Photosensory hypersensitivity of ceh1. a Seeds of P and ceh1 were germinated and grown under continuous red light of various fluence rate $\left(0,0.01,0.1,1,10\right.$, and $\left.50 \mu \mathrm{Em}^{-2} \mathrm{~s}^{-1}\right)$ for 7 days before hypocotyl length measurements. Dark grown (fluence rate 0 ) seedlings were used as control. b $\mathrm{P}$ and ceh1 seeds germinated and grown under short-day (SD; $8 \mathrm{~h}$ light/16 h dark) or continuous white light (CL) for 7 days were

subsequently subjected to hypocotyl length measurements. Data in (a) and (b) are mean \pm SD, each with three biological replicates and 45 seedlings per replicate. Asterisks denote significant differences as determined by a two-tailed Student's $t$ tests with a significance of $P<0.05$

of ceh1/PIF5-OX, a phenotype consistent with their differential growth phenotypes, namely larger ceh1/ PIF5-OX seedlings (Fig. 4b, e and Supplementary Fig. 2c).

This data alludes to high phyB level in ceh1, in part due to reduced PIF4 and 5 transcript levels, and further illustrates a dominant role of PIF5 in MEcPP-mediated regulation of phyB abundance.

Auxin treatment reduces phyB protein abundance in ceh1. The established role of PIF4 and 5 in transcriptional regulation of auxin biosynthetic and signal transduction genes through targeting their promotors $8,18,19,29$, together with our report on MEcPP-mediated reduction of auxin level and auxin transport ${ }^{25}$, prompted us to examine a potential involvement of auxin in regulation of phyB protein abundance in ceh1. Thus, we monitored growth phenotypes, and phyB protein levels of $\mathrm{P}$ and ceh 1 seedlings grown in the absence (mock) and the presence of exogenously applied auxin (Fig. 5a, b). The result corroborate our previous finding ${ }^{25}$, and further establish that auxin induction of hypocotyl growth is in concert with reduced phyB levels in all auxin treated seedlings, most notably in the ceh1 mutant background. This data was further confirmed by genetic approaches using double mutant lines of ceh1 introgressed into auxin receptor mutant tir1-1 (ceh1/tir1-1) known to be deficient in a variety of auxin-regulated growth processes including hypocotyl elongation and normal response to auxin ${ }^{30}$. Subsequently, we examined growth phenotypes and phyB protein abundance in $\mathrm{P}$, ceh1, ceh1/tir1-1, and tir1-1 seedlings grown in the presence and absence of auxin (Fig. 6a, b). This data clearly shows that auxin treatment is notably less effective in promoting hypocotyl growth in ceh1/tir1-1 as compared with the ceh1 mutant. Notably, auxin effect on reducing phyB abundance is attenuated in tir1-1 backgrounds as compared with the respective parent genotypes.

Collectively, these results strongly support the negative feedback regulatory function of auxin in regulating phyB protein abundance.

PhyB protein abundance in ceh1 is CAMTA3-dependent. We have previously established that $\mathrm{MEcPP}$-mediates induction of general stress response genes via activation of calmodulin-binding transcriptional activator 3 (CAMTA3), in a calcium dependent manner ${ }^{24}$. In addition, we previously showed and here 
a
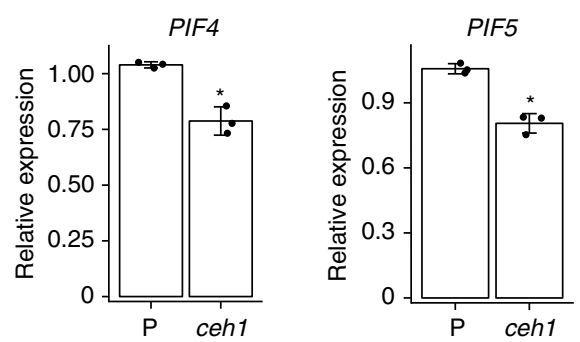

b

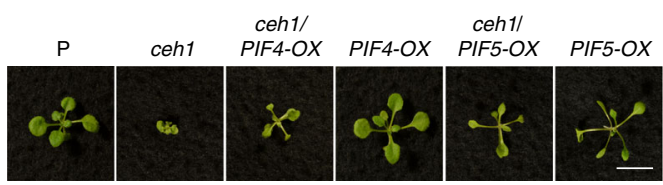

c

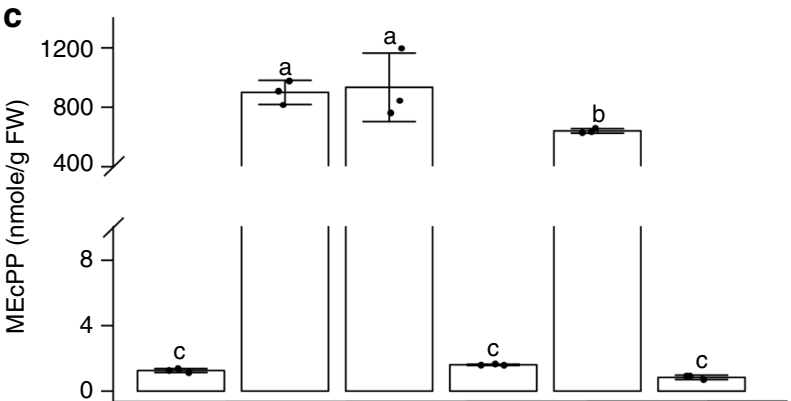

d

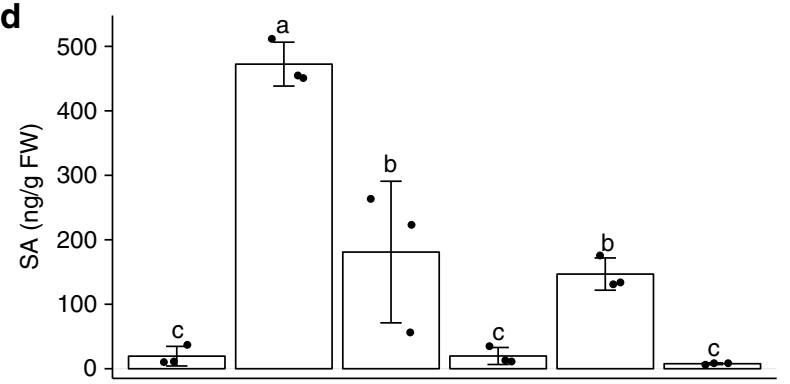

e

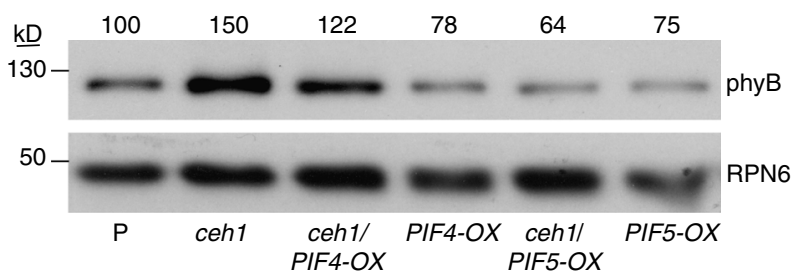

Fig. 4 PIF4 and PIF5 overexpression mimics phyB revertants phenotypes. a Expression levels of PIF4 and PIF5 in P and ceh1 seedlings. Experiments were performed as described in Fig. 2a. Asterisks denote significant differences as determined by a two-tailed Student's $t$ tests with a significance of $P<$ 0.05. b Representative images of 2-week-old P, ceh1, ceh1/PIF4-OX, PIF4OX, ceh1/PIF5-OX, and PIF5-OX seedlings. Scale bar: $1 \mathrm{~cm}$. Levels of MEcPP, with the white line indicating a change of scale on the $Y$ axis (c) and SA (d) in the aforementioned genotypes. Data are mean \pm SD of each genotype with three replicates. Letters above bars indicate significant differences determined by Tukey's HSD method $(P<0.05)$. e Abundance of phyB in total protein extracts of $P$, ceh1, ceh1/PIF4-OX, PIF4-OX, ceh1/PIF5-OX, and PIF5-OX seedlings. Immunoblots were carried out as described in Fig. 2b, with RPN6 as the protein loading control. Numbers above the immunoblot image represent normalized phyB abundance in the genotypes examined relative to $P$ plants a
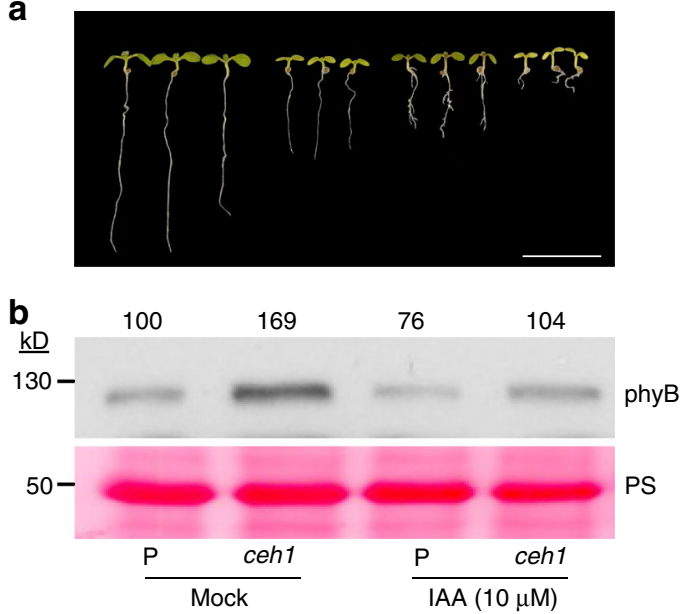

Fig. 5 Auxin treatment reduces phyB protein in ceh1. a Representative images of 7-day-old LD ( $16 \mathrm{~h}$ light/ $8 \mathrm{~h}$ dark) grown $\mathrm{P}$ and ceh1 seedlings in the absence (mock, $0.01 \%$ ethanol) and the presence of IAA. Scale bar: 1 $\mathrm{cm}$. b Abundance of phyB protein in total protein extracts of $P$ and ceh1 seedlings grown in the absence (mock) and presence of IAA. Numbers above the immunoblot image represent normalized phyB abundance in mock and IAA treated seedlings relative to $P$ plants. Ponceau S (PS) staining was used as the loading control

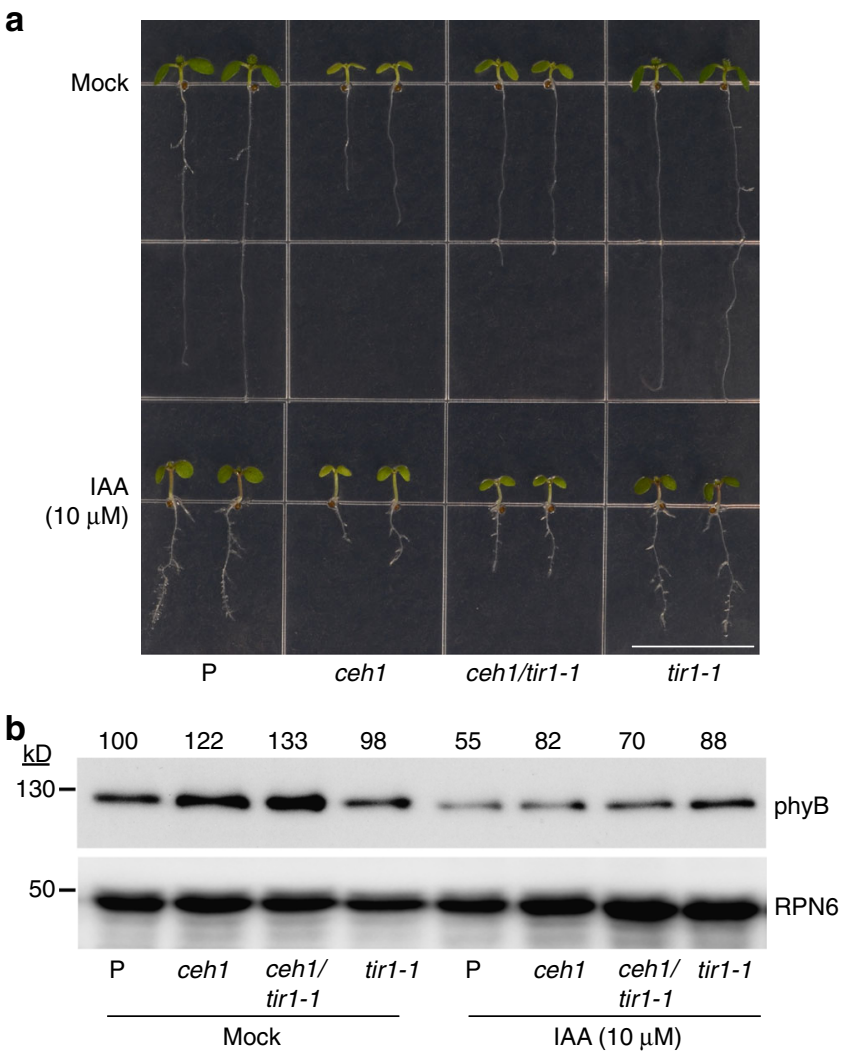

Fig. 6 Auxin regulation of phyB abundance is attenuated in tir1-1. a Representative images of 7-day-old LD (16 h light/8 h dark)grown P, ceh1, ceh1/tir1-1, and tir1-1 seedlings in the absence (mock, $0.01 \%$ ethanol) and the presence of IAA. Scale bar: $1 \mathrm{~cm}$. b Abundance of phyB protein in total protein extracts of $P$, ceh1, ceh1/tir1-1, and tir1-1 seedlings in the absence (mock) and presence of IAA. RPN6 was used as the loading control. Numbers above the immunoblot image represent normalized phyB abundance in the examined genotypes relative to $P$ seedlings 
reconfirmed the partial size recovery of ceh1 dwarf stature in the ceh1/camta3 double mutant lines ${ }^{24}$ (Fig. 7a). This result prompted us to perform RNA seq analyses on camta3 mutant lines in P and ceh 1 mutant backgrounds. Bioinformatics analyses of the data determined that the expression of $\sim 90 \%$ of repressed genes in ceh1 are CAMTA3-dependent, and are significantly overrepresented by genes in light- and auxin-response pathways (Supplementary Fig. 3a and Supplementary Data 1).

This data prompted us to extend the bioinformatics analyses and compare these CAMTA3-dependent genes with those IAA ${ }^{31}$ or PIF $^{32}$ regulated genes (Supplementary Fig. $3 \mathrm{~b}, \mathrm{c}$ and Supplementary Data 2 and 3). Consistent with the GO term analysis (Supplementary Fig. 3a), among the CAMTA3suppressed genes, $\sim 9.6 \%$ and $\sim 10.4 \%$ genes are overrepresented in light- and auxin-response pathways induced by PIF and IAA, respectively. We also compared the microarray dataset of constitutively active allele of phyB $(Y H B)^{33}$, with CAMTA3dependent genes and found that $\sim 17.3 \%$ CAMTA3-induced genes are also upregulated in $Y H B$, and $\sim 8.1 \%$ CAMTA3-suppressed genes are also downregulated in $Y H B$ (Supplementary Fig. 3d, e and Supplementary Data 4 and 5). These data illustrate an overlap between gene regulatory network regulated by $\mathrm{MEcPP}$ and those controlled by CAMTA3, PIF, phyB, and auxin.

This data in concert with our previous observations ${ }^{24}$ followed by reconfirmation of partial recovery of ceh 1 dwarf phenotype in a ceh1/camta 3 double mutant (Fig. 7a) prompted us to examine the likelihood of CAMTA3 involvement in regulation of phyB in the ceh1 background. Initial expression analyses show insignificant differences in the $P H Y B$ transcript levels in examined genotypes ( $\mathrm{P}$, ceh1, ceh1/camta3, and camta3) (Supplementary Fig. 4a). Next, we examined phyB protein abundance in these genotypes (Fig. $7 \mathrm{~b}$ and Supplementary Fig. 4b). Clear reduction of phyB protein abundance in ceh1/camta3 to the basal levels present in $\mathrm{P}$ and camta3 supports the notion of the regulatory function of CAMTA3 in maintaining high phyB protein levels in the ceh1 background.

Given the established dependency of CAMTA3 functionality on $\mathrm{Ca}^{2+}$ together with the proposed function of MEcPP as a rheostat for release of $\mathrm{Ca}^{2+}$ for activation of CAMTA3 ${ }^{24}$, we examined phyB protein abundance in $\mathrm{P}$ and ceh 1 in the presence and absence (mock) of lanthanum chloride $\left(\mathrm{LaCl}_{3} ; \mathrm{Ca}^{2+}\right.$ channel blocker $)^{34}$. The data illustrate reduced phyB protein levels in seedlings treated with this agent most notably in the ceh 1 mutant as compared with the mock treated seedlings (Fig. 7c, d and Supplementary Fig. 4c). The result collectively supports regulatory function of CAMTA3 in stabilizing phyB protein levels in a $\mathrm{Ca}^{2+}$-dependent manner.

Bortezomib stabilizes phyB protein level in ceh1/camta3. To explore mode of CAMTA3 action in stabilizing phyB protein levels, we examined phyB protein levels in LD grown $\mathrm{P}$, ceh1, ceh1/camta3, and camta3 seedlings treated with a $26 \mathrm{~S}$ proteasome inhibitor, bortezomib ${ }^{35}$. The representative blots clearly show higher phyB protein levels in all bortezomib treated seedlings versus the respective mock treated seedlings (Fig. $8 \mathrm{a}-\mathrm{c}$ and Supplementary Fig. 5a-c). It should be noted that both presented blots consistently display higher phyB protein levels in the presence of proteasome inhibitor albeit at different degrees, most likely due to varying bortezomib penetration rate. This increase is most notable in ceh1/camta3 and camta3 bortezomib treated seedlings versus untreated plants. The apparent variations in phyB protein abundance between untreated and mock treated seedlings (Fig. 8a-c and Supplementary Fig. 5a-c) is most likely due to growth conditions requiring transfer of seedlings into liquid media for mock treatment versus a

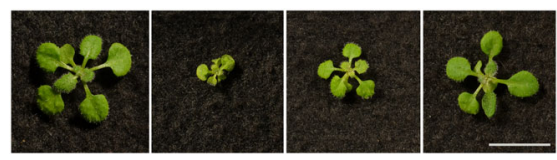

b $_{\mathrm{kD}}$

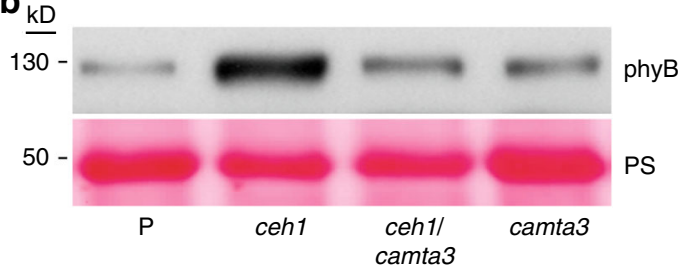

C

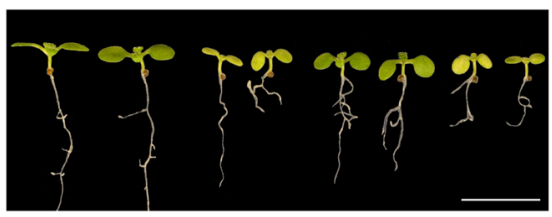

d

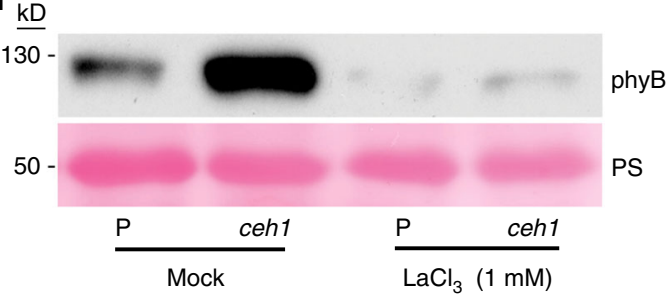

Fig. 7 PhyB protein abundance in ceh1 is CAMTA3- and $\mathrm{Ca}^{2+}$-dependent. Representative images (a) and phyB protein levels (b) in 7-day-old LD (16 h light/8 h dark) grown P, ceh1, ceh1/camta3, and camta3 seedlings.

Representative images (c) and phyB protein levels (d) in 7-day-old $\mathrm{P}$ and ceh1 seedlings in the absence (mock, $0.01 \%$ ethanol) and presence of $\mathrm{LaCl}_{3}$ respectively. Ponceau S (PS) staining was used as loading control. Scale bar: $1 \mathrm{~cm}$

maintaining the untreated seedlings on plates, as described in the Method section.

Collectively, the result strongly supports the notion of CAMTA3 function in stabilizing phyB protein level by impairing proteasome-mediated degradation machinery.

\section{Discussion}

The photoreceptor phyB and the plastidial retrograde signaling metabolite MEcPP are two evolutionarily conserved sensing and signaling components essential for integrity of plants in ever changing environment. The data presented here reveal a functional link between these two essential sensing and signaling pathways and uncovers phyB as component of MEcPP signal transduction pathway, and further identifies MEcPP-mediated multifaceted regulatory network responsible for increased phyB protein abundance and the respective physiological ramifications such as aligning growth and development with stress responses in a constantly changing light environment.

Here, we specifically identify two independent suppressor lines, each with a non-sense mutation in $P H Y B$, displaying a partial reversion of the dwarf phenotype of the ceh1 mutant. In addition, we demonstrate comparable phyB protein in light and dark grown seedlings of constitutively and inducible MEcPPaccumulating lines, as opposed to light-induced reduction of phyB levels in the control line. This is an unexpected finding since 


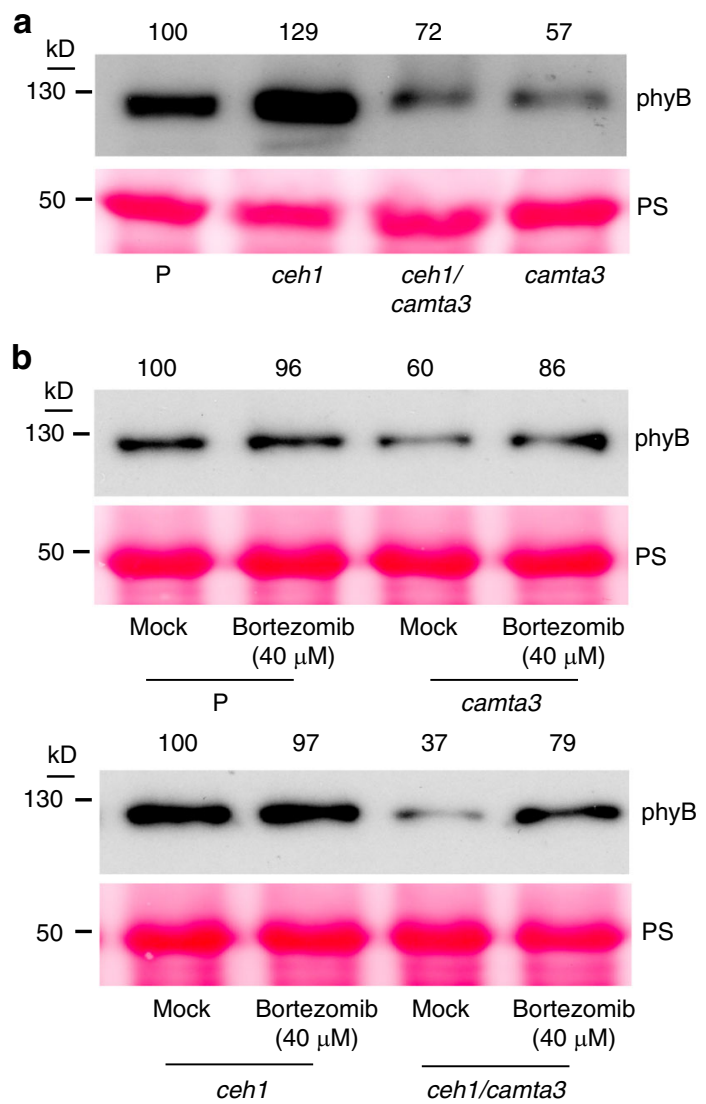

Fig. 8 CAMTA3 stabilizes phyB protein abundance. a phyB protein abundance in nontreated 7-day-old LD grown $\mathrm{P}$, ceh1, ceh1/camta3, and camta3 seedlings. $\mathbf{b}$ phyB protein abundance in 7-day-old LD ( $16 \mathrm{~h}$ light $/ 8 \mathrm{~h}$ dark) grown $\mathrm{P}$, ceh1, ceh1/camta3, and camta3 seedlings in the absence (mock, 0.1\% DMSO) and presence of the $26 \mathrm{~S}$ proteasome inhibitor, bortezomib. Ponceau S (PS) staining was used as loading control. Numbers on top of the immunoblot images represent normalized phyB abundance in various genotypes and in response to bortezomib treatment relative to the P (the top two panels) or ceh1 (the bottom panel) seedlings

light mediates re-localization of cytosolic phyB to nucleus, and subsequent induction of a mutually regulatory intermolecular transaction between phyB and PIF transcription factor leading to rapid degradation of both proteins, and concomitant negative feedback modulation of phyB levels in Arabidopsis ${ }^{36}$. High abundance of phyB in light grown ceh1 seedlings could contribute to stunted phenotype of the mutant as evidenced from earlier reports on the growth retardation of Arabidopsis plants overexpressing $\mathrm{phyB}^{37}$. The role of phyB in regulating growth and development is reported to be through repressing auxin response genes as well as through regulation of both SUR2, a suppressor, and TAA1, an enhancer of IAA biosynthesis ${ }^{38,39}$. Intriguingly, SUR2 (RED1/ATR4) is a cytochrome P450 monooxygenase that moderates the balance between IAA and plant defense compounds, indole glucosinolates ${ }^{40,41}$. This data together with high and selective production of indole glucosinolates in the ceh1 mutant leads to the assumption that MEcPP-mediated induction of this defense metabolite is likely via enhanced abundance of phyB leading to modulation of auxin levels and by extension indole glucosinolates in plants ${ }^{42}$. In fact regulatory function of phyB in plant defense responses is also evident from enhancement of anti-insect resistance via functional integration of $\mathrm{phyB}$ with jasmonates, likely through a conserved hardwire transcriptional network that attenuates growth upon activation of jasmonate signaling ${ }^{43}$. Importantly, reduced SA levels in suppressor lines as well as in ceh1/phyB-9 double mutant compared with the levels in ceh1 background is a clear testimony to the role of phyB in regulating defense hormones.

The next question is the nature of MEcPP-targeted regulatory processes that stabilize phyB levels in light. The schematic model (Fig. 9) depicts MEcPP-mediated regulation of phyB abundance via multiple intersecting pathways, amongst them reduced expression of PIF4 and 5 contributing to phyB stability, in conjunction with reduced auxin known to be regulated by $\mathrm{PIFs}^{8,18,29}$. Auxin is also controlled by MEcPP-mediated reduction of the levels and distribution of this hormone by dual transcriptional and posttranslational regulatory inputs ${ }^{25}$. In addition to the known phyB regulation of auxin, here we establish the reciprocal and negative feedback regulatory function of auxin in adjusting phyB protein abundance as evidenced by reduced phyB levels in seedlings treated exogenously with auxin, and notable attenuation of the response in genotypes in the auxin receptor mutant (tir1-1) background. This data together with earlier reports of auxin regulation by phyB establish a mutually negative, feedback-loop between phyB and auxin.

Importantly, we show CAMTA3-dependent maintenance of high phyB protein abundance. Previously established comparable auxin levels in ceh1 and ceh1/camta3 discard the potential action of CAMTA3 through alteration of auxin levels ${ }^{24}$. As such, we propose that function of CAMTA3 is likely via impairment of proteasome-mediated degradation of the light-activated photoreceptor proteasome COP1-mediated proteolysis of phyB. Indeed, the established phyB degradation mediated by COP1 E3 ligase ${ }^{4}$, and impairment of phyA degradation in the presence of sucrose $e^{45}$, offer a potential link between carbon source and COP1-mediated proteolysis of phyB in cehl.

Pharmacological application of a proteasome inhibitor bortezomib resulting in stabilization of phyB protein levels, most notably in camta 3 mutant backgrounds, strongly supports a direct or indirect function of CAMTA3 in stabilizing phyB protein levels by impairing proteasome-mediated degradation machinery.

In summary, this report unlocks the complexities of plastidial regulated growth-defense tradeoffs and specifically identifies the stress-specific plastidial retrograde signaling metabolite, MEcPP, as an orthogonal regulatory hub of photomorphogenesis through regulation of intersecting pathways of CAMTA3-based transcriptional network and auxin signaling cascade, which collectively control phyB abundance. Delineation of this exquisitely tightly controlled circuitry via plastidial retrograde signaling provides a prime example of biological organization whereby a specific plastidial signal such as MEcPP is transduced into a repertoire of biological responses that collectively regulate photomorphogenesis, a central adaptive strategy critical for securing organismal integrity. Furthermore, this work provides insight into governing rules of complex biological systems and identifies plastids not only as the metabolic hub but also as a center for integration of sensory and signaling networks central to plant adaptive responses to the prevailing environment.

\section{Methods}

Plant materials. Arabidopsis plants used in this study were all in Columbia- 0 background. The control, parent line (P) was transgenic line expressing HPL::LUC construct initially employed for EMS mutagenesis and isolation of ceh1 mutant. tir1-1 (CS3798) was ordered from ABRC, and ceh1/tir1-1 double mutant was generated by crossing the two mutant lines. PIF4-OX and PIF5-OX were gifts from Dr. Peter Quail (University of California, Berkeley).

Suppressor screen. EMS mutagenesis was performed on seeds ( 200 mg) of ceh1 mutant lines. Seeds were treated overnight with $0.2 \%(\mathrm{v} / \mathrm{v})$ ethylmethylsulfonate 


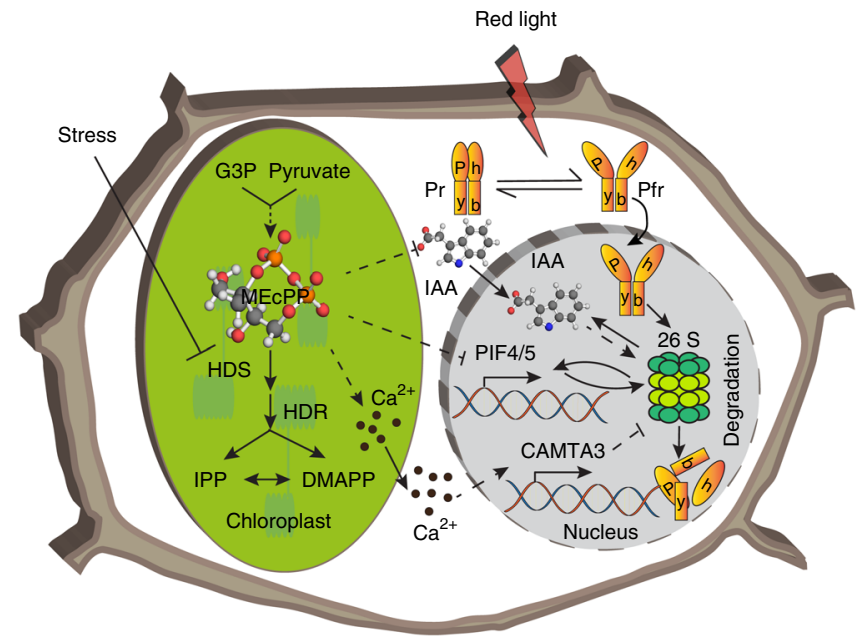

Fig. 9 Orthogonal regulation of phyB protein abundance by MEcPP. Stress interference with HDS enzyme activity and the consequential accumulation of MEcPP transduces intersecting signaling circuitries that regulate abundance of the red-light photoreceptor, phyB, via auxin signaling cascade and $\mathrm{Ca}^{2+}$-dependent CAMTA3-based transcriptional network. The likely function of CAMTA3 is to stabilize phyB protein levels through impairment of proteasome-mediated degradation of the light-activated phyB

(EMS), followed by three washes with $0.1 \mathrm{M}$ sodium thiosulfate and subsequent eight rinses with water. The M1 plants were grown on soil under long-day (LD; $16 \mathrm{~h} \mathrm{light} / 8 \mathrm{~h}$ dark) condition at $22^{\circ} \mathrm{C}$. The M2 seeds were harvested and bulked into 206 pools by pots. Around 100 M2 plants from each pool were screened for mutants with fully or partially bigger size than that of $c e h 1$. The selected M2 plants were grown for two additional generations and only the mutants with inheritable reverted ceh1 growth phenotype were selected, amongst them 41-16 and 56-6 lines. The long hypocotyl and long petiole of these two revertants were reminiscent of the established phyB mutant phenotypes. This led us to directly sequence PHYB and identify nonsense mutations responsible for early termination of coding regions of the two alleles. Complementation studies confirmed phyB as the suppressor of ceh1.

$\mathrm{LaCl}_{3}$ and IAA treatments. Surface-sterilized seeds were planted on half strength Murashige and Skoog medium (1/2 MS: $2.2 \mathrm{~g} / \mathrm{L}$ Murashige and Skoog salts, pH 5.7, and $8 \mathrm{~g} / \mathrm{L}$ agar), stratified at $4{ }^{\circ} \mathrm{C}$ for 3 days, and germinated under $\mathrm{LD}$ for 4 days prior to their transfer onto plates containing 1/2 MS medium together with mock control $\left(0.01 \%\right.$ ethanol), $\mathrm{LaCl}_{3}(1 \mathrm{mM})$, and IAA $(10 \mu \mathrm{M})$ for 3 additional days prior to analyses.

Bortezomib treatment. Surface-sterilized seeds were planted on 1/2 MS plates, stratified at $4{ }^{\circ} \mathrm{C}$ for 3 days, grown under LD for 7 days. These seedlings were then transferred to liquid $1 / 2$ MS media containing mock control (0.1\% DMSO) or bortezomib $(40 \mu \mathrm{M})$ dissolved in $0.1 \%$ DMSO for $8 \mathrm{~h}$ on a shaker in the light chamber ${ }^{35}$.

Light curve of continuous red light. Surface-sterilized seeds were planted on $1 / 2$ MS plates, stratified at $4{ }^{\circ} \mathrm{C}$ for 5 days, and subsequently germinated and grown under continuous red light at various fluence rate for 7 days prior to hypocotyl measurement. Hypocotyl measurement was performed using image J25.

Quantitative RT-PCR. The RNA extraction and data normalization were performed as previously described ${ }^{25}$. Gene-specific primers were designed using QuantPrime q-PCR primer design tool (http://www.quantprime.de/) and listed in Supplementary Table 1. Each experiment was performed with at least three biological and each with three technical replicates.

Protein extraction and immunoblot analyses. Total and nuclear fraction protein extractions were performed according to reported protocol with minor modifications ${ }^{46}$. Specifically, $0.2 \mathrm{~g}$ of 7 -day-old seedlings were collected, ground with liquid nitrogen, homogenized in extraction buffer $(10 \mathrm{mM}$ Hepes, $\mathrm{pH}$ 7.6, $1 \mathrm{M}$ Sucrose, 5 $\mathrm{mM} \mathrm{KCl}, 5 \mathrm{mM} \mathrm{MgCl} 2,5 \mathrm{mM}$ EDTA, $14 \mathrm{mM}$ 2-ME, 0.4\% Triton X-100, $0.4 \mathrm{mM}$ PMSF, $20 \mu \mathrm{M}$ MG132, $20 \mu \mathrm{M}$ MG115, and Proteinase Inhibitor). Crude extract was filtered through Miracloth and saved as total protein. For nuclear fractionation, after filtration, the crude extract was set on ice for $10 \mathrm{~min}$, loaded on top of $15 \%$ percoll and centrifuged at $3000 \times g$ for $10 \mathrm{~min}$ at $4{ }^{\circ} \mathrm{C}$. Upon removal of the supernatant, the pellet was resuspended in extraction buffer followed by the repetition of percoll steps. The re-suspended pellet from this round was saved as nuclear protein. Proteins were separated on 7.5\% SDS-PAGE gel, transferred to PVDF membranes. Blots were probed with anti-phyB monoclonal B1 (1:300) or B1-7 (1:500) primary antibodies obtained from P. Quail lab. The secondary was anti-mouse horseradish peroxidase (HRP) (KPL, catalog no. 074-1806) (1:10,000). Chemiluminescent reactions were performed using the Pierce ECL Western Blotting Substrate following the manufacturer's instructions. The excessive substrate was removed from membranes before placing them between two plastic sheets to develop with X-ray, and subsequently scanned with Epson Perfection V600 Photo Scanner.

SA extraction and analyses. To extract $\mathrm{SA}, 50 \mathrm{mg}$ of flash frozen plant tissue was ground in liquid nitrogen and extracted at $4^{\circ} \mathrm{C}$ with $10 \% \mathrm{mM}$ methanol and $1 \%$ acetic acid containing deuterated SA standard. Extracts were centrifuge twice and $20 \mu \mathrm{l}$ of the clear supernatant was analyzed using a Dionex Ultimate 3000 binary RSLC system coupled to Thermo Q-Exactive Focus mass spectrometer with a heated electro spray ionization source. Sample separation was performed using a AcclaimTM RSLC $120 \mathrm{C} 18$ column $(100 \times 2.1 \mathrm{~mm}$, particle size $2.2 \mu \mathrm{M}$; Thermo Scientific 068982). Gradient elution was acetonitrile containing $0.1 \%$ formic acid followed with water containing $0.1 \%$ formic acid at a flow rate of $200 \mu \mathrm{L} / \mathrm{min}$. The column temperature was maintained at $35^{\circ} \mathrm{C}$. Mass spectra were acquired in negative mode. Retention time and mass transitions was monitored on purified standard and plant matrixes. For relative quantitation, peak area for each compound (MS2; Thermo Trace Finder Software) was normalized to the initial fresh weight mass.

MEcPP extraction and analyses. To extract MEcPP, $50 \mathrm{mg}$ of flash frozen tissue was ground in liquid nitrogen and extracted at $4{ }^{\circ} \mathrm{C}$ with $10 \% \mathrm{mM}$ methanol with $1 \%$ acetic acid, containing $10 \mu \mathrm{M}$ of the deuterated standards. Extract was centrifuged twice and $100 \mu \mathrm{l}$ of the clear supernatant was freeze dried and subsequently reconstituted in $500 \mu \mathrm{l}$ acetonitrile- $10 \mathrm{mM}$ ammonium acetate $(80: 20, \mathrm{v} / \mathrm{v})$. Ten microliters of the resuspended solution was injected to LC-MS system, and analyzed using a Dionex Ultimate 3000 binary RSLC system coupled to Thermo QExactive Focus mass spectrometer with a heated electro spray ionization source. Sample was separated on an Accucore-150-Amide-HILIC column $(150 \times 2.1 \mathrm{~mm}$; particle size $2.6 \mu \mathrm{M}$; Thermo Scientific 16726-152130) with a guard column containing the same column matrix (Thermo Scientific 852-00; 16726-012105). Gradient elution was performed with acetonitrile (A) and $10 \mathrm{mM}$ ammonium acetate $\mathrm{pH} 7.0$ (B). The separation was conducted using the following gradient profile $(t$ (min), \%A, \%B): $(-2,90,10),(0,90,10),(12,30,70),(15,30,70),(16,90,10),(22$ $90,10)$. Flow rate was maintained at $280 \mu \mathrm{L} / \mathrm{min}$. The column was kept at $35^{\circ} \mathrm{C}$. Mass spectra were acquired in negative mode and compound of interest was identified by accurate mass measurements (MS1), retention time and mass transitions of purified standard and plant matrixes.

Sequencing and bioinformatics analysis. RNA-seq libraries were constructed following BrAD-seq method ${ }^{47}$ with six biological replicates. Seventy-five bases of single-end reads of the libraries were sequenced using the Illumina HiSeq 4000 at UC Davis DNA Technologies Core (United States). TopHat2 was utilized to map the reads to A. thaliana genome after trimming adaptors ${ }^{48}$. Subsequently DESeq2 was carried out to count and normalize the mapped reads ${ }^{49}$. Genes with $>2$-fold expression level change relative to control and $P$-value $\leq 0.05$ were selected as differentially expressed genes (DEGs), which was further analyzed using AgriGO (http://bioinfo.cau.edu.cn/agriGO/analysis.php) to identify enriched Gene Ontology (GO) terms base on biological process category.

Statistical analyses. All experiments were performed with at least three biological replicates. Data are mean \pm standard deviation (SD). The analyses were carried out via a two-tailed Student's $t$ tests with a significance of $P<0.05$.

Reporting summary. Further information on research design is available in the Nature Research Reporting Summary linked to this article.

\section{Data availability}

RNA-seq data generated as part of this study has been deposited at the NCBI Sequence Read Archive with accession code PRJNA545720. The overlap between CAMTA3-, IAAPIF-, and YHB-regulated gene lists is provided in Supplementary Data 1-5. The source data underlying Figs. 1(c, d), 2(a-c), 3(a, b), 4(a, c-e), 5(b), 6(b), 7(b, d) and 8(a, b) are provided as Source Data file. The source data underlying Supplementary Figs. 1(a-c), 2(a, b), 4(a-c) and $5(\mathrm{a}-\mathrm{c})$ are provided as Source Data file. Any additional data or biological material that support the findings of this study are available from the corresponding author upon reasonable request.

Received: 16 January 2019 Accepted: 4 June 2019

Published online: 02 July 2019 


\section{References}

1. Devlin, P. F., Patel, S. R. \& Whitelam, G. C. Phytochrome E influences internode elongation and flowering time in Arabidopsis. Plant Cell 10, 1479-1487 (1998).

2. Mathews, S. \& Sharrock, R. A. Phytochrome gene diversity. Plant Cell Environ. 20, 666-671 (1997).

3. Quail, P. H. Photosensory perception and signalling in plant cells: new paradigms? Curr. Opin. Cell Biol. 14, 180-188 (2002).

4. Quail, P. H. et al. Phytochromes: photosensory perception and signaltransduction. Science 268, 675-680 (1995).

5. Nagy, F. \& Schafer, E. Phytochromes control photomorphogenesis by differentially regulated, interacting signaling pathways in higher plants. Annu. Rev. Plant Biol. 53, 329-355 (2002).

6. Leivar, P. \& Quail, P. H. PIFs: pivotal components in a cellular signaling hub. Trends Plant Sci. 16, 19-28 (2011).

7. Rausenberger, J. et al. An integrative model for phytochrome B mediated photomorphogenesis: from protein dynamics to physiology. Plos ONE 5, e10721 (2010).

8. Leivar, P. \& Monte, E. PIFs: systems integrators in plant development. Plant Cell 26, 56-78 (2014).

9. Ni, W. et al. A mutually assured destruction mechanism attenuates light signaling in Arabidopsis. Science 344, 1160-1164 (2014).

10. Casal, J. J. Photoreceptor signaling networks in plant responses to shade. Annu. Rev. Plant Biol. 64, 403-427 (2013).

11. Franklin, K. A. Shade avoidance. New Phytol. 179, 930-944 (2008).

12. Fraser, D. P., Hayes, S. \& Franklin, K. A. Photoreceptor crosstalk in shade avoidance. Curr. Opin. Plant Biol. 33, 1-7 (2016).

13. Chapman, E. J. et al. Hypocotyl transcriptome reveals auxin regulation of growth-promoting genes through GA-dependent and -independent pathways. Plos One 7, e36210 (2012).

14. de Wit, M., Ljung, K. \& Fankhauser, C. Contrasting growth responses in lamina and petiole during neighbor detection depend on differential auxin responsiveness rather than different auxin levels. New Phytol. 208, 198-209 (2015).

15. King, J. J., Stimart, D. P., Fisher, R. H. \& Bleecker, A. B. A mutation altering auxin homeostasis and plant morphology in Arabidopsis. Plant Cell 7, 2023-2037 (1995).

16. Zhao, Y. D. et al. A role for flavin monooxygenase-like enzymes in auxin biosynthesis. Science 291, 306-309 (2001).

17. Zhao, Y. D. et al. Trp-dependent auxin biosynthesis in Arabidopsis: involvement of cytochrome P450s CYP79B2 and CYP79B3. Genes Dev. 16, 3100-3112 (2002).

18. Franklin, K. A. et al. Phytochrome-interacting factor 4 (PIF4) regulates auxin biosynthesis at high temperature. Proc. Natl Acad. Sci. USA 108, 20231-20235 (2011).

19. Nozue, K., Harmer, S. L. \& Maloof, J. N. Genomic analysis of circadian clock-, light-, and growth-correlated genes reveals Phytochrome-Interacting Factor5 as a modulator of auxin signaling in Arabidopsis. Plant Physiol. 156, 357-372 (2011).

20. Sellaro, R., Pacin, M. \& Casal, J. J. Diurnal dependence of growth responses to shade in Arabidopsis: role of hormone, clock, and light signaling. Mol. Plant $\mathbf{5}$, 619-628 (2012).

21. de Souza, A., Wang, J. Z. \& Dehesh, K. Retrograde signals: integrators of interorganellar communication and orchestrators of plant development. Annu. Rev. Plant Biol. 68, 85-108 (2017).

22. Xiao, Y. et al. Retrograde signaling by the plastidial metabolite MEcPP regulates expression of nuclear stress-response genes. Cell 149, 1525-1535 (2012).

23. Lemos, M. et al. The plastidial retrograde signal methyl erythritol cyclopyrophosphate is a regulator of salicylic acid and jasmonic acid crosstalk. J. Exp. Bot. 67, 1557-1566 (2016).

24. Benn, G. et al. Plastidial metabolite MEcPP induces a transcriptionally centered stress-response hub via the transcription factor CAMTA3. Proc. Natl Acad. Sci. USA 113, 8855-8860 (2016).

25. Jiang, J. et al. Interplay of the two ancient metabolites auxin and MEcPP regulates adaptive growth. Nat. Commun. 9, 2262 (2018).

26. Bjornson, M. et al. Integrated omics analyses of retrograde signaling mutant delineate interrelated stress-response strata. Plant J.: Cell Mol. Biol. 91, 70-84 (2017).

27. Chai, T., Zhou, J., Liu, J. \& Xing, D. LSD1 and HY5 antagonistically regulate red light induced-programmed cell death in Arabidopsis. Front Plant Sci. 6, 292 (2015).

28. Leivar, P., Monte, E., Cohn, M. M. \& Quail, P. H. Phytochrome signaling in green Arabidopsis seedlings: impact assessment of a mutually negative phyBPIF feedback loop. Mol. Plant 5, 734-749 (2012).

29. Hornitschek, P. et al. Phytochrome interacting factors 4 and 5 control seedling growth in changing light conditions by directly controlling auxin signaling. Plant J.: Cell Mol. Biol. 71, 699-711 (2012).
30. Ruegger, M. et al. The TIR1 protein of Arabidopsis functions in auxin response and is related to human SKP2 and yeast grrlp. Genes Dev. 12, 198-207 (1998)

31. Nemhauser, J. L., Mockler, T. C. \& Chory, J. Interdependency of brassinosteroid and auxin signaling in Arabidopsis. PLoS Biol. 2, E258 (2004).

32. Zhang, Y. et al. A Quartet of PIF bHLH factors provides a transcriptionally centered signaling hub that regulates seedling morphogenesis through differential expression-patterning of shared target genes in Arabidopsis. PLoS Genet. 9, e1003244 (2013)

33. Jones, M. A., Hu, W., Litthauer, S., Lagarias, J. C. \& Harmer, S. L. A constitutively active allele of phytochrome B maintains circadian robustness in the absence of light. Plant Physiol. 169, 814-825 (2015).

34. Sharma, S. S., Sharma, S. \& Rai, V. K. The effect of EGTA, calcium-channel blockers (lanthanum chloride and nifedipine) and their interaction with abscisic-acid on seed-germination of Brassica-Juncea Cv RLM-198. Ann. Bot.Lond. 70, 295-299 (1992).

35. $\mathrm{Xu}, \mathrm{X}$. et al. Reciprocal proteasome-mediated degradation of PIFs and HFR1 underlies photomorphogenic development in Arabidopsis. Development 144, 1831-1840 (2017).

36. Ni, W. et al. Multisite light-induced phosphorylation of the transcription factor PIF3 is necessary for both its rapid degradation and concomitant negative feedback modulation of photoreceptor phyB levels in Arabidopsis. Plant Cell 25, 2679-2698 (2013).

37. Wagner, D., Tepperman, J. M. \& Quail, P. H. Overexpression of phytochrome $\mathrm{B}$ induces a short hypocotyl phenotype in transgenic Arabidopsis. Plant Cell 3 1275-1288 (1991)

38. Devlin, P. F., Yanovsky, M. J. \& Kay, S. A. A genomic analysis of the shade avoidance response in Arabidopsis. Plant Physiol. 133, 1617-1629 (2003).

39. Halliday, K. J., Martinez-Garcia, J. F. \& Josse, E. M. Integration of light and auxin signaling. Cold Spring Harb. Perspect. Biol. 1, a001586 (2009).

40. Grubb, C. D. \& Abel, S. Glucosinolate metabolism and its control. Trends Plant Sci. 11, 89-100 (2006).

41. Hoecker, U., Toledo-Ortiz, G., Bender, J. \& Quail, P. H. The photomorphogenesis-related mutant red1 is defective in CYP83B1, a red lightinduced gene encoding a cytochrome P450 required for normal auxin homeostasis. Planta 219, 195-200 (2004).

42. Wang, J. Z. et al. Initiation of ER body formation and indole glucosinolate metabolism by the plastidial retrograde signaling metabolite, MEcPP. Mol. Plant 10, 1400-1416 (2017).

43. Campos, M. L. et al. Rewiring of jasmonate and phytochrome B signalling uncouples plant growth-defense tradeoffs. Nat. Commun. 7, 12570 (2016).

44. Jang, I. C., Henriques, R., Seo, H. S., Nagatani, A. \& Chua, N. H. Arabidopsis Phytochrome Interacting Factor proteins promote phytochrome B polyubiquitination by COP1 E3 ligase in the nucleus. Plant Cell 22, 2370-2383 (2010).

45. Debrieux, D., Trevisan, M. \& Fankhauser, C. Conditional involvement of constitutive photomorphogenic1 in the degradation of phytochrome A. Plant Physiol. 161, 2136-2145 (2013).

46. Qiu, Y. et al. Mechanism of early light signaling by the carboxy-terminal output module of Arabidopsis phytochrome B. Nat. Commun. 8, 1905 (2017).

47. Townsley, B. T., Covington, M. F., Ichihashi, Y., Zumstein, K. \& Sinha, N. R. BrAD-seq: Breath Adapter Directional sequencing: a streamlined, ultra-simple and fast library preparation protocol for strand specific mRNA library construction. Front Plant Sci. 6, 366 (2015)

48. Kim, D. et al. TopHat2: accurate alignment of transcriptomes in the presence of insertions, deletions and gene fusions. Genome Biol. 14, R36 (2013).

49. Love, M. I., Huber, W. \& Anders, S. Moderated estimation of fold change and dispersion for RNA-seq data with DESeq2. Genome Biol. 15, 550 (2014).

\section{Acknowledgements}

We would like to thank Dr. Peter Quail (UC Berkeley) for providing phyB antibody and transgenic PIF lines, Dr. Akira Nagatani (Kyoto University) for providing phyB complementation construct, Dr. Meng Chen (UC Riverside) for allowing us to use his light chambers, and Mr. Derrick R. Hicks (University of Washington) for providing IAA and MEcPP structures for our model. We also would like to thank Dr. Jin-Zheng Wang for his comments on the paper. This work was supported by National Science Foundation (NSF) IOS-1036491, NSF IOS-1352478, and National Institutes of Health (NIH) R01GM107311 to K.D.

\section{Author contributions}

J.J. and K.D. designed the study, J.J., L.Z., H.K. and B.D.L.C. performed the experiments, and K.D. wrote the paper. 


\section{Additional information}

Supplementary Information accompanies this paper at https://doi.org/10.1038/s41467019-10867-w.

Competing interests: Authors declare no competing interest.

Reprints and permission information is available online at http://npg.nature.com/ reprintsandpermissions/

Peer review information: Nature Communications thanks Tessa Burch-Smith and other anonymous reviewer(s) for their contribution to the peer review of this work. Peer reviewer reports are available.

Publisher's note: Springer Nature remains neutral with regard to jurisdictional claims in published maps and institutional affiliations. (c) Open Access This article is licensed under a Creative Commons Attribution 4.0 International License, which permits use, sharing, adaptation, distribution and reproduction in any medium or format, as long as you give appropriate credit to the original author(s) and the source, provide a link to the Creative Commons license, and indicate if changes were made. The images or other third party material in this article are included in the article's Creative Commons license, unless indicated otherwise in a credit line to the material. If material is not included in the article's Creative Commons license and your intended use is not permitted by statutory regulation or exceeds the permitted use, you will need to obtain permission directly from the copyright holder. To view a copy of this license, visit http://creativecommons.org/ licenses/by/4.0/.

(C) The Author(s) 2019 